\title{
Reviewer Thank You
}

\begin{abstract}
The Editorial staff of The Journal of Medical Toxicology appreciates all of those who make the success of the journal possible. A medical journal cannot exist without the tireless, and often thankless, work of its reviewers. The list ONLY includes those reviewers registered on our web based program, Editorial Manager, so many who have reviewed may not be on the list. To those reviewers omitted, we apologize, but also thank you very much.
\end{abstract}

Cynthia Aaron
Mohamed Abdel-Rahman
Redouane Abouqal
Terry Adirim
Ritesh Agarwal
Steven Aks
Walter Alarcon
Timothy Albertson
D. Adam Algren
Thomas Arnold
Gavin Arteel
Kavita Babu
Cornelia Baines
Lydia Baltarowich
Theodore Bania
Donald Barceloux
Carola Bardage
Dana Barr
Fermin Barrueto
J. Dave Barry
D. Nicholas Bateman
Stuart Batterman
Frederic Baud
Martin Belson
John Benitez
Michael Beuhler
Steven Bird
G. Randall Bond
Stephen Borron
George Bosse
Eva Boyez
Gokcen Bozdag
Jeffrey Brent
Daniel Brooks
David Brush
Sean Bryant
Alan Buchwald
Nick Buckley
Kurkhart
Murns
Murns
Math

\author{
Michele Burns Ewald \\ Diane Calello \\ Martin Caravati \\ Edward Cetaruk \\ Arthur Chang \\ Lu Chensheng \\ Roger Chou \\ Richard Church \\ Peter Chyka \\ John Cienki \\ Cathleen Clancy \\ Rick Clark \\ Aaron Collins \\ James Courtney \\ Robert Cox \\ Barbara Crouch \\ Kirk Cumpston \\ John Curtis \\ Paul Dargan \\ Cedric Daubin \\ Andrew Dawson \\ H. J. De Silva \\ Kaathleen Delaney \\ Joao Delgado \\ Francis DeRoos \\ Christopher DeWitt \\ James Diaz \\ Jose Diaz-Alcala \\ Ricardo Dinis-Oliveira \\ Robert Dowsett \\ Michael Eddleston \\ Andrew Erdman \\ Mariya Farooqi \\ Miguel Fernandez \\ Jeffrey Fine \\ Mathias Forrester \\ David Gaist \\ Concetta Gardi \\ Ann-Jeannette Geib \\ Robert Geller \\ Richard Geller
}

\author{
Beth Ginsburg \\ Barry Gold \\ Daniel Goldstein \\ Ronald Gots \\ Andis Graudins \\ David Gummin \\ Leon Gussow \\ Jason Hack \\ Alan Hall \\ Christine Haller \\ Fred Harchelroad \\ Martyn Harvey \\ Kennon Heard \\ Robert Hendrickson \\ Fred Henretig \\ James Hillman \\ Michael Hodgman \\ Robert Hoffman \\ Joel Holger \\ Michael Holland \\ Christopher Holstege \\ Mary Ann Howland \\ Oliver Hung \\ Katie Hurlbut \\ Danayal Ibrahim \\ Geoffrey Isbister \\ Kelly Johnson-Arbor \\ Heath Jolliff \\ James Jones \\ Prashant Joshi \\ Bryan Judge \\ Louise Kao \\ Kenneth Katz \\ Ziad Kazzi \\ Anne-Maree Kelly \\ Catherine Kelly \\ William Kerns, II \\ D. Chris Keyes \\ Eun-Joo Kim \\ Paul G. King \\ Mark Kirk
}

\author{
Ron Kirschner \\ Craig Kitchens \\ Kurt Kleinschmidt \\ OR Koch \\ Aleš Kogoj \\ Michael Kosnett \\ Mark Kostic \\ Edward Krenzelok \\ Edwin Kuffner \\ Melisa Lai \\ Eric Lavonas \\ Jerrold Leikin \\ Vivian Leung \\ Howard Levitin \\ Philippe Lheureux \\ Erica Liebelt \\ Ja-Liang Lin \\ Toby Litovitz \\ Heather Long \\ Sara Long \\ J. Lorenc \\ Jeffrey Love \\ Frank Lovecchio \\ Debbie LoVerde \\ Jennifer Lowry \\ Po-Yung Lu \\ Andres Lugo \\ Binh Ly \\ Gerald Maloney \\ Alex Manini \\ Kathy Marquardt \\ Zarko Martinovic \\ Michael Matteucci \\ Fred McDonald \\ Charles McKay \\ Bruno Megarbane \\ William Meggs \\ M. John Mendelsohn \\ Michael Miller \\ Kirk Mills \\ David Morgan
}




Rita Mrvos
Michael Mullins
David Munter
Mark Mycyk
Jamie Nelsen
Lewis Nelson
Adeline Ngo
Jennifer Oakes
Aryn O'Connor
Gerald O'Malley
Kai Osterberg
Kevin Osterhoudt
John Osterloh
Mary Palmer
Manish Patel
Julie Pavlin
Tamas Peredy
Jeanmarie Perrone
Scott Phillips
Anthony Pizon
R. Ponampalam
Laura Power
Laurie Prescott
Roy Purssell

\author{
Michael Reed \\ Arne Reimers \\ Joseph Rella \\ William Richardson \\ Raymond Roberge \\ William Robertson \\ Renee Robinson \\ Gary Rockwood \\ M. G. Roma \\ Ruddy Rose \\ Patricia Rosen \\ Jonathan Rosenson \\ Anna Rouse \\ Anne-Michelle Ruha \\ Steven Salhanick \\ Matthew Salzman \\ Anthony Scalzo \\ Elizabeth Scharman \\ Jay Schauben \\ Joshua Schier \\ James Schmidt \\ Heather Schumann \\ Michael Schwartz \\ David Schwartz
}

Donna Seger
Gilad Segev
Robert Seidner MD
Steven Seifert
Shahin Shadnia
Marco Sivilotti
Carl Skinner
Susan Smolinske
Curtis Snook
Henry Spiller
Andrew Stolbach
Christine Stork
Mark Su
Jeffrey Suchard
Daniel Sudakin
David Tanen
Asim Tarabar
Daniel Teitelbaum
Brigham Temple
Wayne Temple
Milton Tenenbein
Jerry Thomas
Trevonne Thompson
Josef Thundiyil

John Tilelli

Karine Titier

Anthony Tomassoni

Christian Tomaszewski

Stephen Traub

Lauren Trilli

Geoffrey Tucker

Larissa Velez

Rais Vohra

Javier Waksman

Frank Walter

Richard Wang

William Waring

David Watts

Kenneth Whitlow

Ian Whyte

Brandon Wills

Chris Winder

Mary Wittler

Amy Wolkin

David Wood

Alan Woolf

Eduard Zaloshnja

Christina Zhang 